BULL. AUSTRAL. MATH. SOC.

VOL. II (1974), 47-55.

\title{
Approximative compactness and continuity of metric projections
}

\section{B.B. Panda and O.P. Kapoor}

In the paper "Some remarks on approximative compactness", Rev. Roumaine Math. Pures Appl. 9 (1964), I van Singer proved that if $K$ is an approximatively compact Chebyshev set in a metric space, then the metric projection onto $K$ is continuous. The object of this paper is to show that though, in general, the continuity of the metric projection supported by a Chebyshev set does not imply that the set is approximatively compact, it is indeed so in a large class of Banach spaces, including the locally uniformly convex spaces. It is also proved that in such a space $X$ the metric projection onto a Chebyshev set is continuous on a set dense in $X$.

\section{Introduction}

The important concept of the approximative compactness of a set, which was first introduced by Efimov and Stečkin [2], arises quite naturally in the theory of best approximation in normed linear spaces. We recall that a set $K$ in a normed linear space $X$ is called approximatively compact if, for any $x$ in $X$ and any sequence $\left\{g_{n}\right\}$ in $K$, the relation $\left\|x-g_{n}\right\| \rightarrow \inf \{\|x-g\|: g \in K\}$ implies that $\left\{g_{n}\right\}$ is compact in $x$ in the sense that there is a subsequence of $\left\{g_{n}\right\}$ which converges in $k$. Every boundedly compact set is approximatively compact, and in a uniformly convex Banach space every weakly sequentially closed set is approximatively

Received 25 March 1974. The first author was on leave at the IIT Kanpur. 
compact. Singer [8] characterised those spaces which share this property of uniformly convex Banach spaces. In the same paper Singer proved that an approximatively compact Chebyshev set supports a continuous metric projection ([8], Corollary 2). This came as a generalization of various continuity properties of the metric projection given by Klee ([5], Propositions 2.3 and 2.4) and Ky Fan and Glicksberg ([3], Theorem 8). In the present paper we prove that in a large class of Banach spaces which we call "spaces with property $(M)$ " a converse of Singer's result holds: namely, the continuity of the metric projection onto a Chebyshev set $K$ implies the approximative compactness of the set $K$. We also prove that in such a space $X$ the metric projection supported by a Chebyshev set is always continuous on a subset dense in $X$. Again it is shown that a proximal sun in such a space is approximatively compact and hence in a Banach space with property $(M)$ every Chebyshev subspace supports a continuous metric projection.

Throughout this paper we shall denote a real normed linear space by $X$ and its conjugate space by $X^{*}$. Let

$$
B[x, r]=\{y \in X:\|x-y\| \leq r\}, \quad U(X)=\{y \in X:\|y\| \leq 1\},
$$

and

$$
S(X)=\{y \in X:\|y\|=1\} .
$$

The distance from a point $x$ in $X$ to a subset $K$ of $X$ is defined by $d_{K}(x)=\inf \{\|x-y\|: y \in K\}$. The metric projection $P_{K}$ supported by a subset $K$ of $X$ is a mapping defined by

$$
P_{K}(x)=\left\{y \in K:\|x-y\|=d_{K}(x)\right\} \text { for } x \text { in } X .
$$

$K$ is a proximal (respectively Chebyshev) set provided ${ }_{K}(x)$ contains at least (respectively exactly) one point for each $x \in X$. It is called a sum if for each $x \in X$, there is a $v \in P_{K}(x)$ such that $v \in P_{K}(v+\lambda(x-v))$ for every $\lambda \geq 0 \cdot P_{K}$ is said to be upper semicontinuous at $x$ if for every open set $M \subset K$ such that $P_{K}(x) \subset M$, there exists in $M$ an open neighborhood $V$ of $x$ such that $P_{K}(y) \subset M$ for every $y \in V$.

A subgradient of a proper convex function $f$ at a point $x \in X$ is an $x^{*} \in X^{*}$ such that 


$$
f(y) \geq f(x)+x^{\star}(y-x) \text {, for all } y \in X .
$$

In particular, the norm being a proper convex function, an element $x^{*} \in X^{*}$ is a subgradient of the norm at $x \neq \theta \quad(\theta$ will denote the null element of $X)$, if and only if $\left\|x^{*}\right\|=1$ and $x^{*}(x)=\|x\|$.

A normed linear space $X$ is said to have property $(M)$ if the following holds:

For any $x$ in $S(X)$, each sequence $\left\{g_{n}\right\}$ contained in $U(X)$

with $\left\|g_{n}-x\right\| \rightarrow 2$ has a convergent subsequence in $U(X)$.

Clearly every locally uniformly convex normed linear space has property $(M)$. Indeed it can be easily proved that $X$ is locally uniformly convex if and only if it is strictly convex and has property $(M)$. The spaces with property $(M)$ have been discussed in another paper by the authors [7]. Lemma 2 of the present paper contains one geometric property of the unit sphere of such spaces.

\section{The main result}

We now prove our main result, the 'only if' part of which was proved by singer as mentioned above.

THEOREM 1. Let $K$ be a Chebyshev subset of a normed linear space $X$ which has property $(M)$. Then $K$ is approximatively compact if and only if the metric projection $P_{K}$ is continuous on $X$.

For the proof of the theorem we need the following lemma due to Vlasov ([9], Lemma 1), a proof of which is given below for the sake of completeness.

LEMMA 1. Let $X$ be a normed linear space, and let $K$ be a Chebyshev subset of $X$. If $x \in X$ is a point of continuity of the metric projection $P_{K}$ then

$$
\lim _{t \rightarrow 0} \frac{d_{K}\left(x+t\left(x-P_{K}(x)\right)\right)-d_{K}(x)}{t}=d_{K}(x) \text {. }
$$

Proof of Lemma 1. Let $z \in X$ and let $\left\{t_{n}\right\}$ be any sequence of positive real numbers converging to zero. Let $\phi_{n}$ be a subgradient of the 
norm at $x-P_{K}\left(x+t n^{z)}\right.$. Then for all $n \geq 1$,

$$
\phi_{n}\left(x-P_{K}\left(x+t_{n} z\right)\right)=\left\|x-P_{K}\left(x+t_{n} z\right)\right\|,
$$

and

$$
\begin{aligned}
\left\|x+t_{n} z-P_{K}\left(x+t_{n} z\right)\right\| & \geq\left\|x-P_{K}\left(x+t_{n} z\right)\right\|+\phi_{n}\left(t_{n} z\right) \\
& \geq d_{K}(x)+\phi_{n}\left(t_{n}^{z}\right),
\end{aligned}
$$

so that

$$
d_{K}\left(x+t_{n} z\right)-d_{K}(x) \geq \phi_{n}\left(t_{n}^{z)}\right.
$$

Again let $\phi_{n}^{\prime}$ be a subgradient of the norm at $x+t_{n} z-P_{K}(x)$. Then for all $n \geq 1$,

$$
\phi_{n}^{\prime}\left(x+t_{n} z-P_{K}(x)\right)=\left\|x+t_{n} z-P_{K}(x)\right\|
$$

and

$$
\begin{aligned}
\left\|x-P_{K}(x)\right\| & \geq\left\|x+t_{n} z-P_{K}(x)\right\|-\phi_{n}^{\prime}\left(t_{n}^{z)}\right. \\
& \geq d_{K}\left(x+t_{n} z\right)-\phi_{n}^{\prime}\left(t_{n}^{z}\right)
\end{aligned}
$$

so that

$$
d_{K}\left(x+t_{n} z\right)-d_{K}(x) \leq \phi_{n}^{\prime}\left(t_{n} z\right)
$$

Now let $\phi$ and $\phi^{\prime}$ be any weak*-cluster points of $\left\{\phi_{n}\right\}$ and $\left\{\phi_{n}^{\prime}\right\}$ respectively. Then by (1) and (3) and the continuity of $P_{K}$ we have

$$
\phi\left(x-P_{K}(x)\right)=\left\|x-P_{K}(x)\right\|=\phi^{\prime}\left(x-P_{K}(x)\right)
$$

and thus $\phi$ and $\phi^{\prime}$ are subgradients of the norm at $x-P_{K}(x)$. From (2) and (4) we get

$$
\phi_{n}(z) \leq \frac{d_{K}\left(x+t_{n} z\right)-d_{K}(x)}{t_{n}} \leq \phi_{n}^{\prime}(z) .
$$

Putting $z=x-P_{K}(x)$ and taking account of (5) we conclude that

$$
\lim _{t \rightarrow 0^{+}} \frac{d_{K}\left(x+t\left(x-P_{K}(x)\right)\right)-d_{K}(x)}{t}=d_{K}(x) \text {. }
$$


In the same way we can prove that

$$
\lim _{t \rightarrow 0^{-}} \frac{d_{K}\left(x+t\left(x-P_{K}(x)\right)\right)-d_{K}(x)}{t}=d_{K}(x),
$$

and hence the result is proved.

Proof of Theorem 1. Let $\left\{g_{n}\right\} \subset K, x \in X \sim K$ and $\left\|x-g_{n}\right\| \rightarrow d_{K}(x)$. Suppose that $P_{K}$ is continuous. Set $t_{n}=\left(\left\|x-g_{n}\right\|-d_{K}(x)\right)^{\frac{1}{2}}$. Clearly $t_{n}$ tends to zero through nonnegative values. We can assume without loss of generality that $t_{n}>0$ by passing on to a subsequence, if neçessary. Let $\phi_{n}$ be a subgradient of the norm at $x+t_{n}\left(x-P_{K}(x)\right)-g_{n}$ so that

$$
\phi_{n}\left(x+t_{n}\left(x-P_{K}(x)\right)-g_{n}\right)=\left\|x+t_{n}\left(x-P_{K}(x)\right)-g_{n}\right\| \text {, }
$$

and

$$
\begin{aligned}
\left\|x-g_{n}\right\| & \geq\left\|x+t_{n}\left(x-P_{K}(x)\right)-g_{n}\right\|-t_{n} \phi_{n}\left(x-P_{K}(x)\right) \\
& \geq d_{K}\left(x+t_{n}\left(x-P_{K}(x)\right)\right)-t_{n} \phi_{n}\left(x-P_{K}(x)\right) .
\end{aligned}
$$

Hence

$$
\frac{d_{K}\left(x+t_{n}\left(x-P_{K}(x)\right)\right)-d_{K}(x)}{t_{n}} \leq t_{n}+\phi_{n}\left(x-P_{K}(x)\right) \text {. }
$$

Applying Lemma 1 to $(7)$ we see that $\left\|x-P_{K}(x)\right\| \leq \phi_{0}\left(x-P_{K}(x)\right)$, where $\phi_{0}$ is any weak*-cluster point of the sequence $\left\{\phi_{n}\right\}$. This implies that $\phi_{0}\left(x-P_{K}(x)\right)=\left\|x-P_{K}(x)\right\|$. We assume without loss of generality that $\left\{\phi_{n}\right\}$ converges to $\phi_{0}$ in the weak*-topology. Now set

$$
z_{n}=\frac{x+t_{n}\left(x-P_{K}(x)\right)-g_{n}}{\left\|x+t_{n}\left(x-P_{K}(x)\right)-g_{n}\right\|} \quad \text { and } \quad z=\frac{x-P_{K}(x)}{\left\|x-P_{K}(x)\right\|} .
$$

Then

$$
2=\lim _{n \rightarrow \infty} \phi_{n}\left(z_{n}+z\right) \leq \underset{n \rightarrow \infty}{\lim \inf }\left\|z_{n}+z\right\| \leq \underset{n \rightarrow \infty}{\lim \sup }\left\|z_{n}+z\right\| \leq 2
$$

and hence $\left\|z_{n}+z\right\| \rightarrow 2$ as $n \rightarrow \infty$. Since $X$ has property $(M)$, the sequence $\left\{z_{n}\right\}$ has a convergent subsequence. Moreover, 


$$
\lim _{n \rightarrow \infty}\left\|x+t_{n}\left(x-P_{K}(x)\right)-g_{n}\right\|=\lim _{n \rightarrow \infty}\left\|x-g_{n}\right\|=d_{K}(x), x \in X \sim K,
$$

and hence $\left\{g_{n}\right\}$ also has a convergent subsequence.

The converse is well known and holds even in a metric space setting (see $[8]$ ).

The following example shows that, in general, continuity of the metric projection does not imply that the Chebyshev set is approximatively compact.

EXAMPLE. Let $X$ be the dual space of the Banach space constructed by Klee [6] by suitably renorming $z^{2}$. Lambert (unpublished) has shown that in the space $X$ the metric projection $P_{K}$ supported by any Chebyshev subspace $K$ of $X$ is continuous. However, $X$ does not satisfy the Efimov-Stečkin property and hence contains a closed hyperplane $X$ which is not approximatively compact (Singer, [8], Theorem 3). Since $X$ is strictly convex and reflexive, $K$ is a Chebyshev subspace and thus supports a continuous metric projection. The space $X$ of course would not have property $(M)$ (see also Holmes [4], p. 165).

\section{Some further results}

We give next a geometric property of the unit sphere of spaces with property $(M)$. This property will be used to obtain some more results about continuity behaviour of metric projections.

LEMMA 2. Let $X$ be a normed linear space with property $(M)$. Let $x$ be a nonzero element of $U(X)$, and let

$$
K_{n}=B[x, 1-\|x\|+1 / n] \sim \operatorname{int} U(X) .
$$

Then each sequence $\left\{g_{n}\right\}$ with $g_{n} \in K_{n}$ has a convergent subsequence in $X \sim \operatorname{int} U(X)$.

Proof. From

$$
1-\|x\| \leq\left\|g_{n}\right\|-\|x\| \leq\left\|x-g_{n}\right\| \leq 1-\|x\|+1 / n,
$$

it follows that $\left\|g_{n}\right\|+1$ as $n \rightarrow \infty$. Now there exists $\psi_{n} \in S\left(X^{*}\right)$ such that $\psi_{n}\left(g_{n}\right)=\left\|g_{n}\right\|$ and hence 


$$
\left\|g_{n}\right\|=\psi_{n}\left(g_{n}-x+x\right) \leq\left\|x-g_{n}\right\|+\psi_{n}(x) .
$$

If $\psi_{0}$ is any weak*-cluster point of the sequence $\left\{\psi_{n}\right\}$ then from (8) we get $1 \leq 1-\|x\|+\psi_{0}(x)$; that is, $\|x\| \leq \psi_{0}(x)$. As $\left\|\psi_{0}\right\| \leq \underset{n \rightarrow \infty}{\lim \inf }\left\|\psi_{n}\right\|=1$, this implies that $\psi_{0}(x)=\|x\|$ and $\left\|\psi_{0}\right\|=1$. Now set $z_{n}=g_{n} /\left\|g_{n}\right\|$ and $z=x /\|x\|$. Then taking a subsequence $\left\{\psi_{n_{i}}\right\}$ such that $\psi_{n_{i}} \rightarrow \psi_{0}$ in the weak*-topology, we get

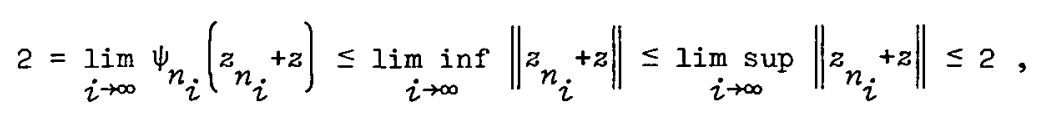

and thus $\lim _{i \rightarrow \infty}\left\|z_{n_{i}}+z\right\|=2$. As $X$ has property $(M)$ the sequence. $\left\{z_{n_{i}}\right\}$ has a convergent subsequence in $U(X)$. Hence $\left\{g_{n_{i}}\right\}$ has a convergent subsequence in $X \sim \operatorname{int} U(X)$. This in turn implies that the original sequence has a convergent subsequence in $X \sim \operatorname{int} U(X)$. This proves the lemma.

THEOREM 2. Let $X$ be a space with property $(M)$, and let $K$ be a proximal sun in $X$. Then

(i) $K$ is approximatively compact, and

(ii) $P_{K}$ is upper semicontinuous on $X$.

Proof. Let $\left\{g_{m}\right\} \subset K$ be a sequence such that $\left\|g_{m}-x\right\| \rightarrow d_{K}(x)$. Since $K$ is a sun, there is a point $y \in P_{K}(x)$ such that all points on the ray $\overrightarrow{y x}$ are projected on $y$. Take $z_{0}=x+\lambda_{0}(x-y)$ where $\lambda_{0}>0$ and set

$$
K_{n}=B[x,\|x-y\|+1 / n] \sim \operatorname{int} B\left[z_{0},\left\|z_{0}-y\right\|\right]
$$

Since $\left\|g_{m}-x\right\| \rightarrow\|x-y\|=d_{K}(x)$, there exists for each $n$ a $g_{m_{n}}$ such that $g_{m_{n}} \in K_{n}$. By Lemma 2, there exists a subsequence of $\left\{g_{m_{n}}\right\}$ which is convergent in $K \quad(K$ is closed $)$. Thus $K$ is approximatively compact. 
Since the metric projection supported by an approximatively compact set is upper semi-continuous (Singer [8], Theorem 1), (i) implies (ii).

REMARK. As a consequence of Theorem 2 all proximal subspaces in a space $X$ with property $(M)$ are approximatively compact. Thus all reflexive spaces having property $(M)$ satisfy the Efimov-Steckin property (see Singer [8]). However, it is known that the converse is not true (see [7].).

In the following we use Lemma 2 to obtain another result about the continuity behaviour of the metric projection onto a Chebyshev set which may not be a sun.

THEOREM 3. Let $K$ be a Chebyshev set in: a normed Iinear space $X$ with property $(M)$. Then $P_{K}$ is continuous on a subset dense in $X$.

Proof. Let $x \in X \sim K$ and let $z \neq x$ be any point on the line segment between $x$ and $P_{K}(x)$; that is, $z=\lambda x+(1-\lambda) P_{K}(x)$ for $0 \leq \lambda<1$. Let $\left\{z_{n}\right\}$ be any sequence converging to $z$. It can be easily proved that $\left\|P_{K}\left(z_{n}\right)-z\right\| \rightarrow\left\|P_{K}(z)-z\right\|$. Now an application of Lemma 2 in the manner it was done in Theorem 2 shows that the sequence ${ }_{K}\left(P_{K}\left(z_{n}\right)\right\}$ is compact in $K$. As $K$ is Chebyshev this means that $P_{K}\left(z_{n}\right) \rightarrow P_{K}(z)$ in norm. On the other hand, if $x \in K$ then $P_{K}(x)=x$ and hence $P_{K}$ is continuous on $K$. Combining these two we get the required result.

The assumption. in Theorem 3 that $\cdot K$ is Chebyshev can be relaxed provided the norm in $X$ has some additional properties.

THEOREM 4. Let $K$ be either

(a) a closed subset of a uniformly convex Banach space, or

(b) a proximal subset of a locally uniformly convex Banach space. Then there exists a subset $G$ dense in $X$ such that the restriction of the metric projection $P_{K}$ to the set $G$ is singlevalued and continuous.

Proof. First, let $K$ be a closed subset of a uniformly convex Banach space. Then by a result of Edelstein [1], there exists a subset $D$ dense in $\dot{X}$ such that every point in $D$ admits a nearest point in $K$. The 
union $G$ of all sets $\left\{\lambda x+(1-\lambda) y: 0 \leq \lambda<1, x \in D, y \in P_{K}(x)\right\}$ is clearly dense in $X$. Moreover, every element of $G$ admits a unique nearest point in $K$. An application of Lemma 2 in the way it was done in Theorem 3 yields the required result.

In the case of $(b)$ the set $D$ is the whole space $X$ and the proof is the same. Hence the theorem is proved.

\section{References}

[1] M. Edelstein, "On nearest points of sets in uniformly convex Banach spaces", J. London Math. Soc. 43 (1968), 375-377.

[2] N.V. Efimov and S.B. Stečkin, "Approximative compactness and Čebyšev sets", Soviet Math. Dokl. 2 (1961), 1226-1228.

[3] Ky Fan and Irving Glicksberg, "Some geometric properties of the spheres in a normed linear space", Duke Math. J. 25 (1958), 553-568.

[4] Richard B. Holmes, A course on optimization and best approximation (Lecture Notes in Mathematics, 257. Springer-Verlag, Berlin, Heidelberg, New York, 1972).

[5] Victor Klee, "Convexity of Chebyshev sets", Math. Ann. 142 (1961), 292-304.

[6] V. Klee, "Two renorming constructions related to a question of Anselone", Studia Math. 33 (1969), 231-242.

[7] B.B. Panda and O.P. Kapoor, "A generalization of local uniform convexity of the norm", J. Math. Anal. Appl. (to appear).

[8] Ivan Singer, "Some remarks on approximative compactness", Rev. Roumaine Math. Pures App 2.9 (1964), 167-177.

[9] L.P. Vlasov, "On Čebyšev sets", Soviet Math. Dokl. 8 (1967), 401-404.

Department of Mathematics,

Regional Engineering College,

Rourkela, Orissa,

India.
Department of Mathematics, Indian Institute of Technology, Kanpur, Kanpur, India. 